\title{
Select toxic metals status of pregnant women with history of pregnancy complications in Benin City, South-South Nigeria.
}

\section{*11OTEBHI, GE; OSADOLOR HB}

\author{
Department of Medical Laboratory Science, School of Basic Medical Sciences, University of Benin, Benin City, \\ Nigeria \\ Correspondence author Email: godwinoteb@gmail.com \\ Email: humphreyosadolor@uniben.edu
}

\begin{abstract}
Toxic metals are part of the most important groups of environmental pollutants that can bind to vital cellular components and interfere with their functions via inhalation, foods, water etc. The serum levels of toxic metals (lead, mercury, cadmium and arsenic) in pregnant women with history of pregnancy complications, pregnant women without history of pregnancy complication and non-pregnant women in Benin City, South - South Nigeria was investigated in this paper, with total of 135 healthy women comprising of 45 pregnant women with history of previous pregnancy complications, 45 pregnant women without history of pregnancy complications and 45 non-pregnant women without history of pregnancy complications (controls). Some demographic characteristics and $4 \mathrm{ml}$ of blood samples were obtained from each subject for the analysis of lead, mercury, cadmium and arsenic by standard methods. Pregnant women with history of pregnancy complications recorded a highly significant increase in the toxic metal (lead) mean value of $25.81 \mu \mathrm{g} / \mathrm{dl}$ as against $23.70 \mu \mathrm{g} / \mathrm{dl}$ for pregnant women without history of pregnancy complications and $11.23 \mu \mathrm{g} / \mathrm{dl}$ for non-pregnant (control) women without history of pregnancy complications as well as significant increases in the mean values of other toxic metals (mercury, cadmium and arsenic) compared with controls ( $\mathrm{p}<0.001)$. The selected toxic metals $(\mathrm{Pb}, \mathrm{Hg}, \mathrm{Cd}$ and $\mathrm{As}$ ) may be involved in the development of pregnancy complications among pregnant women in Benin City, South-South Nigeria. Lead in particular may pose threat to mothers and fetuses as its mean values in the two groups of pregnant women were well above normal. (C) JASEM
\end{abstract}

http://dx.doi.org/10.4314/jasem.v20i1.1

KEYWORDS: Toxic metals, pregnant women, History of pregnancy complications.

\section{Introduction}

Pregnancy, which is vital in the course of a woman's life, is a period during which there is high sensitivity to toxic substances. Complications of pregnancy on the other hand are reproductive health issues or problems that occur during pregnancy and may involve the mother's health, the child's health or both. Exposures to toxic metals by pregnant women have been an under-recognized health issues in developing countries such as Nigeria despite the well documented effects of toxic metals, particularly in the South-South region of Nigeria where there is currently no report about the effects of toxic metals on pregnancy outcomes.

Toxic metals and poor maternal diet in pregnancy are example of environmental factors that can impact negatively on the human reproductive system. Studies in animals revealed that the increased uptake of lead, cadmium and other toxic metals interfered with the normal pregnancy course and result in abortion among other physiological dysfunctions (Ajayi et al 2012). In addition, the special route of exposure that is endogenous calls for a great concern as many metals can mobilize from accumulated organs such as bones to the blood stream during pregnancy after period of exposures (Semczuk et al 2001). These metals has the propensity to cross the fetal membrane and potentially harm the fetus and since no level has been proven safe during pregnancy for many toxic metals, focus is now on low-dose exposures that may cause sub clinical problems (Hackley et al 2003).

In a systematic analysis for the Global Burden of Disease, it was reported that complications of pregnancy resulted in 293000 deaths from 37,700 deaths in 1990 and the common causes are maternal bleeding, complications of abortion, birth defects which are majorly influenced by age, multiple pregnancy and environmental toxicant such as lead, mercury and tobacco, etc (GBD 2013).

One of the main mechanisms by which the toxic metals act is by the generation of free radicals reactive oxygen species (ROS) and reactive nitrogen species (RNS), that may resort in oxidative stress which has been reported to influence the female reproductive system adversely (Agarwal et al 2004).

This study was aimed at investigating the select toxic metals- lead, mercury, cadmium and arsenic status in pregnant women with history of pregnancy complications in Benin City, South South Nigeria. 


\section{MATERIALS AND METHODS}

Study Area: The study was done in Benin City, the capital and largest city of Edo State in South - South Nigeria, between January and October 2015. Benin City, the leading home of arts and culture in Nigeria is situated on a branch of the Benin river within the rain forest zone of the South South region of Nigeria. The city is estimated to have 1,125,058 people(Nigeria population update 2015). The site of the study was Central Hospital Benin, a referral hospital situated at the city centre.

Study Population / Subjects: A total of 135 apparently healthy women comprising of 45 pregnant women with one or more history of previous pregnancy complications (such as maternal bleeding, complications of abortion, severe anaemia, placenta previa, gestational diabetes, birth defects etc) and 45 pregnant women without any history of previous pregnancy complications (all within the 1st and into the 2nd trimesters) and 45 non-pregnant women without history of pregnancy complication were recruited for this study after obtaining verbal informed consent and ethical clearance from the Edo State Ministry of Health and Central Hospital authority. The pregnant women were aged between $21-35$ years and within the gestational age of $0-20$ weeks recruited from the Antenatal Clinic of Central Hospital, Benin City. All the women were matched for age and social economic status. Women who were 36 years and above, those with symptoms of diseases such as fever, and increased blood pressure, those already on nutritional supplements and those with multiple pregnancies were excluded from this study. Some demographic and basic health indices were obtained from the subjects through direct questioning / measurement and the use of ANC cards and case notes of pregnant subjects. All the subjects were subjected to the same instruments and method of measurement.

Sample Collection / Preparation: About $4 \mathrm{ml}$ of veinous blood from the antecubital vein was collected from the subjects using sterile, disposable syringes into sterile heparinized sample containers, mixed and kept temporary in the refrigerator $\left(2-8^{\circ} \mathrm{C}\right)$ for the measurement of blood toxic metal - (lead, mercury, cadmium and arsenic) levels.

Acid Digestion: Prior to measurement about $2 \mathrm{ml}$ of whole blood samples were transferred to pre-labelled digestive tubes and $4 \mathrm{ml}$ of concentrated nitric acid added. The samples were placed on a hot plate in the

\section{RESULTS AND DISCUSSION}

Table 1 shows some demographic and basic health indices of pregnant and non-pregnant women with status of complications enrolled in the study. As indicated, participants were matched for age and socio-economic status. In addition some basic health fume chamber and subsequently heated to just below boiling point. The samples were digested slowly for about 3 hours. When the volume had reduced to about $1 / 3,2 \mathrm{ml}$ of $30 \%$ hydrogen peroxide solution was added. The samples were then evaporated slowly, residues dissolved in $1 \%$ nitric acid and filtered to provide sample solutions ready to be aspirated into AAS or ICP-MS.

Biochemical Measurements: Analysis were done using Electrothermal Atomic Absorption Spectrophotometer (Peckin Elmer Analysts 800, Norwalk USA) adopting the method of Olmedo et al,((2010) for the analysis of lead, cadmium and arsenic and inductively coupled plasma mass spectrophotometer (ICP-MS) (Agilent 7500, Norwalk USA) by adopting the method of Fong et al,((2007) for the analysis of blood mercury.

Procedures for Lead, Cadmium and Arsenic Analysis: Lead, cadmium and arsenic were analyzed with Electrothermal Atomic Absorption Spectrophotometer (Peckin Elmer Analysts 800, Norwalk USA) adopting the method of Olmedo et al,((2010) as below:

1The hollow cathode lamp specific for each metal was inserted. The wavelength was adjusted appropriately with respect to the metal under investigation.; The instrument was standardized and calibrated with standard blank and standard for each metal; An aliquot $(\mathbf{2 0} \boldsymbol{\mu l})$ of the prepared sample was injected directly into the graphite furnace. The concentration of analyte under investigation was displayed on the screen after a run time of about 4 minutes.

Procedure for Mercury Analysis: Mercury was determined by inductively coupled plasma mass spectrometer (Agilent 7500, Norwalk, USA) by adopting the method of Fong et al (2007) as below:

The instrument was standardized and calibrated with standard blank and mercury standard. Aliquot of $\mathbf{2 0 \mu l}$ of the prepared sample was aspirated into the quartz spray chamber. The result data was displayed on the screen after the run and was downloaded to Microsoft Excel sheet.

Statistical Analysis: Values were recorded as mean and standard error of mean. Comparison of mean was by one-way analysis of variance (ANOVA) followed by student t-test.

indices including history of previous pregnancy complications were indicated.

Table 2 shows the levels of toxic metals in the pregnant and non-pregnant women with complication status. In comparison, toxic metals levels in pregnant 
women with history of pregnancy complications (PWWHC) were significantly higher than values obtained in pregnant women without history of pregnancy complications (PWWTHC) and non- pregnant women without history of pregnancy complications (NPWWTHC) $(p<0.001)$. A similar trend was observed in the toxic metal levels between PWWTHC and NPWWTHC

- Table 1:Some demographic and basic health indices of pregnant and non-pregnant women with status of complications enrolled in the study

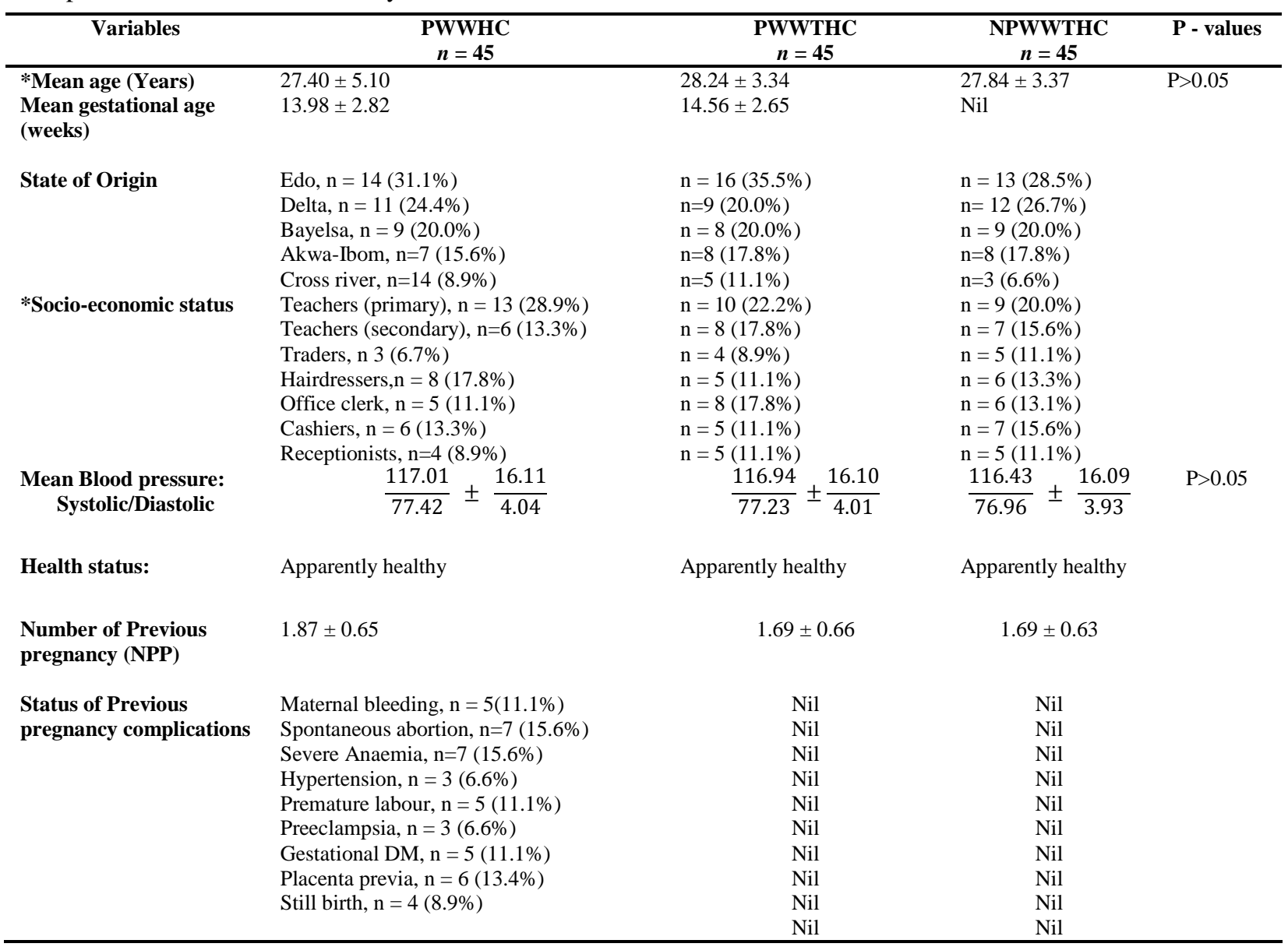

Note:

PWWHC = Pregnant women with history of pregnancy complications

PWWTHC = Pregnant women without history of pregnancy complications

NPWWTCH $=$ Non-pregnant women without history of pregnancy complications

* Participant were matched for Age and socio-economic status

Table 2 Comparison of Levels of Toxic Metals in Pregnant and Non-pregnant Women with Status of Complications in Benin City, South-south Nigeria

\begin{tabular}{|c|c|c|c|c|c|}
\hline Parameters & $\begin{array}{l}\text { Pregnant women With } \\
\text { Complications }\end{array}$ & $\begin{array}{l}\text { Pregnant women } \\
\text { Without } \\
\text { Complications }\end{array}$ & $\begin{array}{l}\text { Non Pregnant women } \\
\text { Without Complications }\end{array}$ & P-value & Significance \\
\hline $\begin{array}{l}P b(\mu g / d l) \\
H g(\mu g / l) \\
C d(\mu g / l)\end{array}$ & $\begin{array}{c}25.81^{A} \pm 0.06 \\
0.23^{A} \pm 0.00 \\
0.89^{A} \pm 0.01\end{array}$ & $\begin{array}{c}23.70^{B} \pm 0.09 \\
0.21^{B} \pm 0.00 \\
0.83^{B} \pm 0.00\end{array}$ & $\begin{array}{c}11.23^{C} \pm 0.15 \\
0.10^{C} \pm 0.00 \\
0.39^{C} \pm 0.00\end{array}$ & $\begin{array}{l}P<0.001 \\
P<0.001 \\
P<0.001\end{array}$ & $\begin{array}{l}\text { Highly Significant } \\
\text { Highly Significant } \\
\text { Highly Significant }\end{array}$ \\
\hline$A s(\mu g / l)$ & $0.18^{A} \pm 0.00$ & $0.17^{B} \pm 0.00$ & $0.08^{C} \pm 0.00$ & $P<0.001$ & Highly Significant \\
\hline
\end{tabular}

Note:

Values in Mean \pm Standard Error of Mean

Superscripts (A, B, C) indicate values with and without statistical differences

This present study was designed to determine the blood levels of selected toxic metals in pregnant women with history of pregnancy complications in Benin City (a densely populated city with increased 
burden from environmental pollution), within the South-South region of Nigeria; which might contribute additional information on the causes of pregnancy complications in the South-South region of Nigeria and also aid in its management strategies.

Pregnancy itself is an important and unique period of a woman's life during which there is high sensitivity to toxic substances.

In this study, we observed a highly significant increase $(\rho<0.001)$ in the blood toxic metals (lead, mercury, cadmium and arsenic) levels in pregnant women with history of pregnancy complications compared with women who are also pregnant but are without any history of pregnancy complications and with non-pregnant women that are without any history of pregnancy complications. These findings have been shown in many other studies from different countries (Coulam et al., 1996 and Apostolic et al., 2011) and also partly in the western region of Nigeria, Ibadan, where similar increases were observed to be associated with spontaneous abortion (Ajayiet al., 2012).

Heavy metals generally have been shown to be one of the most important groups of environmental pollutants having blood reference ranges that differ from country to country and from region to region due to geographical location, soil content, intake from diets and bioavailability (Nwagha et al 2011).

In this study, we recorded highly significant mean level of lead $(\mathrm{P}<0.001)$ well above $10 \mu \mathrm{g} / \mathrm{dl}$ (the United States CDC's recommended cut off level for blood lead level in children and pregnant women), in pregnant women with history of pregnancy complications (PWWHC) and in pregnant women that are without any history of pregnancy complications (PWWTHC) 25.81 \pm 0.06 SEM $(\mu \mathrm{g} / \mathrm{dl}$ ) and $23.70 \pm 0.09 \mathrm{SEM} \mu \mathrm{g} / \mathrm{dl}$ respectively as against $11.23 \pm 0.15 \mathrm{SEM}(\mu \mathrm{g} / \mathrm{dl})$ for non-pregnant women without any history of pregnancy complications (NPWWTHC).

The maximum tolerable blood lead level (BLL) for women of child bearing age recommended by WHO (1980) is $30 \mu \mathrm{g} / \mathrm{dl}$ and no more than $50 \%$ of a population blood lead values should exceed $20 \mu \mathrm{g} / \mathrm{dl}$. The US Centre for Disease Control cut off level for raised BLL for children and pregnant women is $10 \mu \mathrm{g} / \mathrm{dl}$ (CDC 1991).

Maternal BLLs approximately $10 \mu \mathrm{g} / \mathrm{dl}$ have been linked to increased risk of pregnancy hypertension, spontaneous abortion and reduced offspring nuerobehavioural development. (Bellinger 2005). Although the damage is greatest at higher level of accumulation, no level have been found to be safe (Hackleyet al 2003).
The total body content of lead does not produce any feed-back mechanism for its absorption (Demichale 1984). The mean biological life of lead is about 30 days after which majority $(70-95 \%)$ of it is accumulated within the mineralizing tissue like teeth and long bones, with the elimination half-life of 5 10 years (Domichale 1984). But during pregnancy or times of calcium stress, stores of lead deposited in bones overtime are mobilized into the blood stream. The association of increased blood lead levels with pregnancy complications is well documented from various studies in different countries even at recommended levels (Hackley et al., 2003 and Moshen 2014). The highly significant increase in the blood lead mean values of pregnant women with history of pregnancy complications, when compared with the pregnant and non-pregnant women that are without any history of pregnancy complications found in this study may be associated with the development of some of the previous pregnancy complications recorded among these subjects.

The lead data from this studies may suggest that apparent toxic impact of lead could be expected in the case subjects since it has been documented that higher levels of maternal exposure of $>25 \mu \mathrm{g} / \mathrm{dl}$, appear to reduce fertility and increase the risks of spontaneous abortion and preterm delivery. (Owago et al., 2009).

Two theories about the possible pathways of the mechanism of lead toxicity have been described (Vaziri et al., 2007). First, lead can induce excessive production of Reactive Oxygen Species (ROS) which disrupt collagen being a primary target for ROS. This cause the membrane to lose its elasticity leading to premature rupture of membrane with its associated risks. Secondly, balance in reproductive hormones is necessary for the appropriate progress during the course of pregnancy. Lead can disrupt reproductive hormones. Estrogen and progesterone secretion can be affected by lead via increase in luteinizing hormone and follicle stimulating hormone $(\mathrm{Ng}$ et al 1991).

There have been a number of studies examining the association of paternal exposure to mercury and an increased risk for spontaneous miscarriage (Cordier $e t$ al., 1991). In another study, women occupationally exposed to elemental mercury vapour had more spontaneous abortions, still births and congenital malformation and irregular, painful or hemorrhagic menstrual disorders than a control group of women not exposed to mercury.

In this study, there was a highly significant increase $(\rho<0.001)$ in the mean blood mercury level of pregnant women with history of pregnancy complications compared with the pregnant and nonpregnant women that are without history of pregnancy complications. Mercury is a toxic heavy 
metal which is widely dispersed in nature. Most human exposures to mercury is caused by outgassing of mercury from dental amalgam, ingestion of contaminated fish or occupational exposure (WHO, 1991). On entry the body, mercury vapour has great affinity for sulfhydryl groups and binds to sulphurcontaining amino acids throughout the body. Metallic mercury passes easily through the blood brain barrier and also deposit readily in the placenta and fetal tissues (Roland, 1992). The highly significant increase of blood levels of mercury might have contributed to the development of pregnancy complications in the cases.

Mercury in all forms poisons cellular functions by altering the tertiary and quaternary structure of proteins and by binding with sulfhydryl and selenohydryl groups. It is also believed to interfere with DNA transcription and protein synthesis (Roland 1992).

Also in this study, we observed a highly significant increase $(\rho<0.001)$, in the mean blood levels of cadmium and arsenic in pregnant women with history of pregnancy complications compared to pregnant and non-pregnant women without history of pregnancy complications. Cadmium and arsenic are extremely toxic metals which have no known necessary function in the body. Cadmium toxicity commonly arises from eating of contaminated food stuffs and processed food products such as rice and wheat grown in soil contaminated by sewage sludge, super phosphate fertilizer and irrigation water. there is increasing evidence between maternal cadmium exposure and adverse pregnancy outcomes such as reduced size at birth and preterm delivery (Nishigo et al., 2002).

Cadmium toxicity is increasing for several reasons, one of which is primarily zinc deficiency in many commonly eating foods. Zinc which is protective against cadmium is being increasingly deficient in the soil and consequently in foods. Food processing and eating of refined food further reduces zinc intake. Cadmium is also known to displace zinc in many metallo-enzymes thereby impairing the functions of zinc. Cadmium concentrates in the kidney, liver and various other organs and is considered more toxic than lead and mercury(Nishigo et al.,2002).

Arsenic has been suggested to be a reproductive and developmental toxicant in humans, investigations have shown the association between Arsenic and a number of pregnancy outcomes such as still birth, spontaneous abortion, perinatal death, size at birth and structural abnormalities. Arsenic and its methylated metabolites are accumulated in placenta and fetal tissues and subsequently exert toxic effects. This may result in fetal loss (Von-Ehrenstein 2006).
The mechanism of arsenic toxicity is centered on the disruption of ATP production. At the level of the citric-acid cycle, arsenic inhibit pyruvate dehydrogenase and by competing with phosphate, it uncouples oxidative phosphoylation, thereby inhibiting energy-linked reduction of $\mathrm{NAD}^{+}$, mitochondrial respiration and ATP synthesis. Hydrogen peroxide production is also increased which might form reactive oxygen species and therefore oxidative stress (Hughes 2002).

The findings of significantly increased toxic metal levels in pregnant women with history of pregnancy complications in this study, is consistent with the findings of Agarwal et al., 2004, Lamphear 2005 and Neeti 2012.

Conclusion: Our results revealed that blood toxic metal levels in women during pregnancy were significantly raised compared to non-pregnant women, especially the blood lead levels (BLL) as all the pregnant women enrolled in this study recorded a value well above $10 \mu \mathrm{g} / \mathrm{dl}$, which was considered in 1991 to be the bench mark for intervention according to the United States Centre for Disease Control and Prevention. These findings indicate that there are probably many unidentified cases of lead poisoning as the control subjects (non-pregnant women) also recorded values above $10 \mu \mathrm{g} / \mathrm{dl}$ for lead. Clearly, this work is suggestive of a deeper investigations in order to identify the actual sources of lead exposure among the citizenry especially our pregnant mothers. This study may therefore serve as a wake-up call on the State governments within the South South geopolitical zone in Nigeria and perhaps the government of Nigeria as a whole to implement programmes aimed at reducing the blood lead levels in reproductive age women so as to minimize the exposures of our pregnant mothers to lead toxicity thereby adverting possible negative pregnancy outcomes. These issues as well as determining the reference or permissible values of toxic metals for our environment especially the South South region, endowed with lots of water ways and densely populated cities with high vehicular traffics, needs to be addressed in future studies.

\section{REFERENCES}

Afal, A. and Wiener S. W. (2014): Metal Toxicity. Medicine Medscape 81(4): 338-347.

Agarwal A., and Allamaneni S. (2004) Role of free radicals in female reproductive diseases and assisted reproduction. Reprod. Biom. Online 9: $338-347$.

Ajayi, O. D., Charles-Davis, M. A. and Avinola, O. G. (2012): Progesterone, selected heavy metals and micronutrients in pregnant Nigeria women 
with history of recurrent spontaneous abortion. African Health Science, 12(2): 153 - 158.

Apostolic P. and catalane S. (2001): Metal Ions affecting reproduction and development. Metal ions life science 8: 263 - 303.

Bellinger, D. C. (2005): Teratogen update: Lead and pregnancy. Birth defects research part A. Clinical and Molecular Teratology. 73(6): 409 420.

CDC (1991): Preventing lead poisoning in young children. A statement by the Centres for Disease Control Atlanta. GA:Centres for Disease Control.

Cordier, S, Deplan F., Mandereau L, and Heman D. (1991): Paternal exposure to mercury and spontaneous abortions. Br. J. Ind. Med. 48: 375381.

Coulam C. B., Stephen M., Stern J. J. and Clerk D. A. (1996) Immunotherapy for recurrent pregnancy loss: Analysis of results from clinical trials. Am. J. Reprod. Immumol. 35: $\quad 352-359$.

Demichele, S. J. (1984): Nutrition of lead. Comp. Biochem. Physiol. 74: 407 - 408.

Fong, B. M., Lee, T. S. and Tan, S. (2007). Determination of mercury in whole blood and urine by inductively coupled plasma mass spectrometry. J. Anal. Toxicol. 31(5): $281-282$.

GBD (2013) Mortality and causes of death, a stematic analysis for the global burden of disease study

Lancet10 (14) 1990 - 2013.

Hackley B, and Katz-Jacobson A. (2003): Lead poisoning in pregnancy: A case study with implications for midwives. J. Midwifery Women's Health. 48: 30 - 38.

Hughes, M. P. (2002): Arsenic toxicity and potential mechanisms of action. Toxicol Lett 133(1): $1-16$.

Lamphear B. P. Vorhees C. V.; and Bellinger D. C. (2005) Protecting Children from Environmental Toxins. Plos Medicine 2 (3) 61.

Mohsen V. (2014): Effects of lead and other metals on pregnancy outcomes. Tuntendo medical journal 21(5): $65-171$.

Neeti K. and Prakash T., (2013): Effect of heavy metal poison during pregnancy. Int. Res. J. Environmental Sci.2: 88 - 92.
Ng. T.P., Gohft H. and Ng, Y. I. (1991): Male endocrine functions in workers with moderate exposure to lead. Br. J. Ind. Med. 48: 485 - 491.

Nigeria Population update (2015). Nishijo M., Nakagawa H., Honda R., Tenebe K., Saito S. and Tetanishi H. (2002): Effects of maternal exposure to cadmium on pregnancy outcome and breast milk. Occup. Envron. Med. 59: 394 396.

Nwagha, U. I., Ogbodo, S. O., Nwogu-Ikojo, E. E., Ibegbu, D. M., Ejezele, F. E. et al., (2011). Copper and selenium status of healthy pregnant women in Enugu south eastern Nigeria.

Nigerian Journal of Clinical Practice 14(4): 408 -412 .

Olmedo P., Pla A., Hernadez A. F., Lopez-Guanid O., Rodingo L. and Gil F (2010) : Electrothermal Atomization - Atomic Absorption Spectrometry (ET-AAS), Analytical Clinical Acts 659: 60 - 67.

Owago J. O. Murungi J., and Lang'at-Thoruwa C. (2009): A Survey of blood lead level in pregnant women attending two public prenatal clinics in Nairobi city, Kenya. J. Am. Sci. 5 (3): $41-51$.

Roland, A. S. (1992) Reproductive effects of mercury vapour. Found Appl. Toxicol. 19: 326 - 329.

Semczuk, M. and Semczuk-Sikora A. (2001): New data on toxic metal intoxication $(\mathrm{Cd} . \mathrm{Pb}$ and $\mathrm{Hg}$ in particular) and $\mathrm{Mg}$. status during pregnancy. Med. Sci. Monit. 7: 332 - 340.

Vaziri N. D. and Khan M. (2003): Interplay of reactive oxygen species and nitric oxide in the pathogenesis of experimental lead -induced hypertension. Clin. Exp. Pharmacol.physiol $34: 920-925$.

Von-Ehrenstein, O. S. (2006): Pregnancy outcomes, Infant mortality and arsenic in drinking water in West Bengal, India, Am. J. Epidemisr 163(7): $662-669$.

World Health Organization (1991): Inorganic mercury environmental Health Criteria 118 in international program in Chemical Safety. WHO Geneva, Switzerland.

WHO (1980): Recommended health based limits in occupational exposure to heavy metals in Technical report series No. 647. (air quality guidelines for Europe) Copenhagen. World Health Organization Regional Office for Europe. Pp $200-209$. 